REVISIONES

\title{
Metabolismo mineral óseo durante la gestación y efectos sobre la masa ósea de la madre
}

\author{
Bone mineral metabolism during gestation and its effects on maternal bone mass
}

\author{
Luis Vidal ${ }^{1,2}$, Maritza Vidal ${ }^{2}$, Santiago Cabrera ${ }^{3,4}$, Eduardo Ortega ${ }^{2}$, Vicente Santiváñez ${ }^{5}$, \\ Jorge Polo ${ }^{1}$, Jorge Barnaby ${ }^{6}$, Rolando Vargas ${ }^{7}$, Angélica del Castillo ${ }^{8}$ \\ ${ }^{1}$ Hospital Departamental María Auxiliadora. Lima, Perú. \\ ${ }^{2}$ Centro Diagnóstico de la Osteoporosis y Enfermedades Reumáticas. Lima, Perú. \\ ${ }^{3}$ Servicio Materno Fetal, Hospital Nacional Docente San Bartolomé. Lima, Perú. \\ ${ }^{4}$ Facultad de Medicina, Universidad Nacional Mayor de San Marcos. Lima, Perú. \\ ${ }^{5}$ Clínica Anglo-Americana. Lima, Perú. \\ ${ }^{6}$ Centro Nacional de Alimentación y Nutrición, Instituto Nacional de Salud. Lima, Perú. \\ ${ }^{7}$ Departamento de Medicina de la Universidad Nacional de Piura. Piura, Perú. \\ ${ }^{8}$ Clínica San Pablo. Lima, Perú.
}

\section{Resumen}

La gestación y lactancia son periodos de alta demanda de calcio, para el crecimiento fetal y para la producción de leche materna. Durante la gestación, la madre transfiere al feto 25 a $30 \mathrm{~g}$ de calcio y durante la lactancia se secretan 200 a 240 mg de calcio diario en la leche materna. Durante la gestación, los mecanismos adaptativos más importantes son el incremento en la absorción de calcio y el incremento del recambio óseo materno; durante la lactancia, hay tendencia a conservar más calcio a nivel renal, pero la desmineralización temporal del esqueleto materno parece ser el mecanismo más importante. Los estudios que emplean absorciometría dual de rayos X (DXA) son escasos, por el riesgo de radiación materna y fetal; pero, con otras técnicas, como el ultrasonido cuantitativo, se ha descrito una pérdida de masa ósea durante la gestación predominantemente dependiente del hueso trabecular. Mediante marcadores bioquímicos del remodelamiento óseo se ha demostrado que los suplementos de calcio reducen la tasa de remodelamiento durante el embarazo y parecen tener efecto benéfico sobre la pérdida ósea materna. Estudios longitudinales que emplean ultrasonido cuantitativo también han encontrado una disminución de la pérdida ósea, entre las mujeres que recibieron aporte o suplemento adecuado de calcio durante la gestación. Palabras clave: Densidad mineral ósea; gestación; lactancia materna; calcio.

\begin{abstract}
Pregnancy and lactation are periods of high calcium demand for skeletal growth and maternal milk production. Approximately $25-30 \mathrm{~g}$ of calcium are transferred to the fetus during pregnancy, and breast-feeding mothers secrete $200-240 \mathrm{mg} /$ day of calcium in breast milk every day. During pregnancy, major physiologic adaptations include increased both calcium intestinal absorption and rate of maternal bone turnover; during lactation, there is a contribution of renal calcium conservation, but temporary maternal bone demineralization is the main mechanism to meet calcium requirements. Data on bone mineral density assessed by dual energy x-ray absortiometry (DXA) are sparse mainly due to concern about potential risk of radiation exposure to the fetus. Other radiation-free techniques like quantitative ultrasound have been used to assess maternal bone mass changes during pregnancy. Maternal bone loss mainly depending on trabecular areas during pregnancy has been described. Calcium supplements reduce maternal skeletal-bone turnover as evaluated by bone resorption markers during pregnancy and seem to have beneficial effects on maternal bone loss. Longitudinal studies with repeated measurements of quantitative ultrasound during pregnancy have found decrease in bone loss in pregnant women with adequate calcium intake or supplementation.
\end{abstract}

Key words: Bone density; pregnancy; breast feeding; calcium.

\section{Introducción}

Durante la vida de la mujer, la gestación y lactancia son dos etapas que requieren de diferentes adaptaciones fisiológicas, dentro de las cuales se incluyen cambios en el metabolismo mineral óseo, en respuesta a la necesidad de calcio para cubrir las demandas del feto en crecimiento y el requerimiento adicional de calcio para la leche materna, durante el periodo de lactancia.
Los reguladores más importantes de la homeostasis del calcio son la paratohormona, la calcitonina y la 1 , 25 dihidroxivitamina $\mathrm{D}_{3}\left[1,25(\mathrm{OH})_{2}\right.$ $\mathrm{D}_{3}$ ], las cuales se complementan para mantener las concentraciones de calcio sérico dentro de sus rangos normales, a través de tres mecanismos principales: ${ }^{(1)}$ incrementar la absorción intestinal del calcio; ${ }^{(2)}$ estimular la reabsorción renal de calcio; y, ${ }^{(3)}$ movilizar calcio hacia la sangre a partir del hueso ${ }^{(1)}$. El estrés adicional, generado por el esqueleto fe- tal en desarrollo y por el requerimiento de calcio en la leche materna durante la lactancia, puede traer como consecuencia cambios en la densidad mineral ósea de la madre, lo cual parece ser dependiente del estatus nutricional de la madre y del aporte de calcio durante estas etapas de la vida.

Durante la gestación, las adaptaciones fisiológicas más importantes son el incremento de la absorción intestinal del calcio y el incremento en la tasa de 
recambio óseo materno; mientras que, durante la lactancia hay una tendencia a una mayor conservación de calcio a nivel renal; pero, el mecanismo más importante parece ser la desmineralización ósea materna temporal ${ }^{(2)}$.

A lo largo del presente artículo revisaremos con mayor detalle los cambios en el metabolismo mineral en las gestantes y los mecanismos de adaptación requeridos para cubrir las nuevas necesidades fisiológicas impuestas durante esta etapa de la vida, así como, sus repercusiones sobre el feto y la masa ósea de la madre.

\section{Adaptaciones durante la gestación}

En la gestación, el feto es totalmente dependiente de la madre para adquirir minerales, como calcio, fósforo y magnesio, los cuales se transportan activamente en contra de gradiente de concentración a través de la placenta a la circulación fetal; y, en las últimas semanas de gestación, las concentraciones séricas de calcio, fósforo y magnesio en el feto son incluso mayores que las de la madre ${ }^{(3)}$.

A lo largo de todo el periodo de gestación se requiere de aproximadamente 30 a 35 gramos de calcio de la madre destinados al desarrollo del esqueleto fetal, de los cuales $80 \%$ es transferido al feto a través de la placenta, durante el último trimestre de gestación. Debido a que el esqueleto fetal se mineraliza rápidamente después de la segunda mitad del embarazo y alcanza su tasa máxima durante el tercer trimestre, la necesidad de calcio se eleva de $50 \mathrm{mg} /$ día, en la $20^{\mathrm{a}}$ semana, a $330 \mathrm{mg} /$ día, en la $35^{\text {a }}$ semana de gestación. Este requerimiento elevado de calcio hace necesario una serie de adaptaciones en la madre destinadas a cumplir la demanda adicional de este mineral de parte del esqueleto fetal ${ }^{(4)}$.

Durante la gestación, uno de los mecanismos más importantes para suplir la necesidad de calcio adicional es el incremento temprano en la tasa de absorción intestinal de este mineral, mediada por un incremento en la 1, 25 dihidroxivitamina $\mathrm{D}_{3}$. Este incremento es independiente de los cambios en la paratohormona y proviene principalmente de los riñones de la madre, aunque puede haber cierta contribución de la placenta, decidua y riñones fetales ${ }^{(5)}$.

\section{Minerales y hormonas que regulan el calcio}

A pesar de que puede encontrarse una disminución del calcio sérico total, el calcio iónico, que representa la fracción fisiológicamente más importante del calcio, permanece constante durante toda la gestación. El dosaje del calcio iónico en la gestante es la medición más importante y la que debe ser solicitada si es que existe alguna duda acerca del valor real del calcio en cualquier momento de la gestación. En cambio, el calcio sérico total disminuye de manera secundaria a la disminución de albúmina sérica, debida a la expansión del volumen intravascular y hemodilución durante el embarazo ${ }^{(6)}$.

Los estudios iniciales sobre el metabolismo del calcio en gestantes mostraron una disminución del calcio sérico total durante este periodo (suma del calcio ionizado, calcio en complejos y la fracción unida a albúmina), lo que fue considerado como una prueba de que el feto extraía calcio materno; originó el concepto erróneo de que la gestación se asociaba con un estado de 'hiperparatiroidismo secundario' en la madre. Sin embargo, posteriormente se demostró que la caída en el calcio sérico total se debía a la disminución en la albúmina sérica por la expansión del volumen intravascular y hemodilución durante la gestación, que traía como consecuencia la disminución de la fracción del calcio unido a albúmina ${ }^{(7)}$.

A pesar de que anteriormente se informaba sobre niveles elevados de paratohormona en la segunda mitad de la gestación, actualmente está claro que la paratohormona sérica se mantiene dentro de valores en rango normal bajo durante el embarazo. La explicación a esta discrepancia es que las primeras determinaciones de paratohormona sérica aplicadas a la clínica, en la década del 60, se basaban en métodos de radioinmunoanálisis (RIA) que usaban un solo anticuerpo contra la región carboxiterminal o la región media de la hormona; así mismo, dosaban diferentes y múltiples fracciones de la paratohormona, la mayoría de estas biológicamente inactivas ${ }^{(8,9)}$. A partir de 1987, se introdujo los métodos inmunorradiométricos (IRMA), basados en 2 anticuerpos, dirigidos contra las fracciones aminoterminal y carboxiterminal, denominados 'medición en sándwich' o 'paratohormona intacta', que valoran la fracción biológicamente activa de la hormona intacta; estos métodos son ampliamente empleados en clínica e investigación al momento actual ${ }^{(10)}$.

Con estos métodos modernos se ha demostrado que la paratohormona sérica disminuye a un rango normal bajo durante el primer trimestre del embarazo, aproximadamente $10 \%$ a $30 \%$ de la media del valor normal en la no gestante, lo cual puede representar una supresión modesta de la glándula paratiroidea al inicio del embarazo. Sin embargo, los niveles de paratohormona sérica se van incrementando, hasta llegar al nivel promedio normal al final de la gestación. En algunos grupos de mujeres con una ingesta muy pobre de calcio se ha encontrado que esta elevación de la PTH al final del embarazo puede superar los valores normales, como un mecanismo para evitar la pérdida renal de calcio ${ }^{(11,12)}$.

Los niveles de $1,25(\mathrm{OH})_{2} \mathrm{D}_{3}$ se encuentran elevados durante el embarazo y las concentraciones se incrementan en alrededor del $50 \%$ a $100 \%$ durante el segundo trimestre de gestación y en cerca de $100 \%$ durante el tercer trimestre ${ }^{(13)}$. Este incremento es independiente de la paratohormona y parece depender más bien de la sobrerregulación de la 1- $\alpha$-hidroxilasa materna, a nivel renal, debido al efecto del péptido relacionado a la paratohormona (PTHrp). A pesar que la placenta y los riñones fetales pueden producir $1,25(\mathrm{OH})_{2} \mathrm{D}_{3}$, la proporción más importante parece provenir de los riñones maternos.

Durante la gestación, también se incrementan los valores del péptido relacionado a la hormona paratiroidea (PTHrp), el cual proviene de la placenta en etapas tempranas del embarazo y en fases avanzadas de la glándulas paratiroideas del feto ${ }^{(14)}$. La PTHrp podría contribuir a elevar la $1,25(\mathrm{OH})_{2} \mathrm{D}_{3}$ y a suprimir 
a la paratohormona, además de otras acciones probables, como favorecer la resorción ósea materna y la regulación del transporte de calcio de la placenta al feto. Adicionalmente, su porción carboxiterminal, denominada 'osteostatina', tiene efecto inhibidor del osteoclasto y podría más bien proteger el esqueleto materno ${ }^{(15)}$. El PTHrp tiene además importantes funciones en la regulación del desarrollo del hueso endocondral y en el desarrollo del esqueleto fetal ${ }^{(16)}$.

\section{Absorción intestinal y excreción renal de calcio durante la gestación}

El incremento en la absorción de calcio intestinal parece ser el mecanismo mayor para compensar el elevado requerimiento de calcio durante la gestación, y está relacionado con el aumento de la $1,25(\mathrm{OH})_{2} \mathrm{D}_{3}$, con el subsecuente incremento de calbindina ${ }_{9 \mathrm{k}}-\mathrm{D}$ y de otras proteínas que favorecen el transporte de calcio a nivel intestinal. El incremento en la absorción intestinal de calcio es proporcionalmente similar en gestantes adultas y adolescentes, aunque estas últimas no hayan alcanzado su pico de masa ósea ${ }^{(17)}$. Se calcula que la fracción de calcio absorbido se incrementa en 50 a $56 \%$ en el segundo trimestre y $54 \%$ a $62 \%$ en el tercer trimestre ${ }^{(13)}$.

El incremento temprano de la absorción del calcio durante la gestación permite su almacenamiento adecuado en el esqueleto fetal. Si se asume una ingesta diaria de $1000 \mathrm{mg}$ de calcio elemento y en base a una absorción fraccional de 33\%, el incremento en la absorción de calcio estaría resultando en un incremento de 165 a $205 \mathrm{mg}$ de calcio al día destinados al feto. Este calcio teóricamente debería, al menos en parte, mantener el requerimiento de alrededor de $330 \mathrm{mg}$ al día que requiere el feto en las últimas semanas de gestación ${ }^{(1)}$.

La excreción renal de calcio se encuentra incrementada, probablemente, como resultado del aumento en la tasa de filtración glomerular, así como del incremento en la absorción intestinal de calcio. La cantidad de calcio perdido en la orina se calcula entre 80 y $136 \mathrm{mg}$ por día y representa una pérdida que

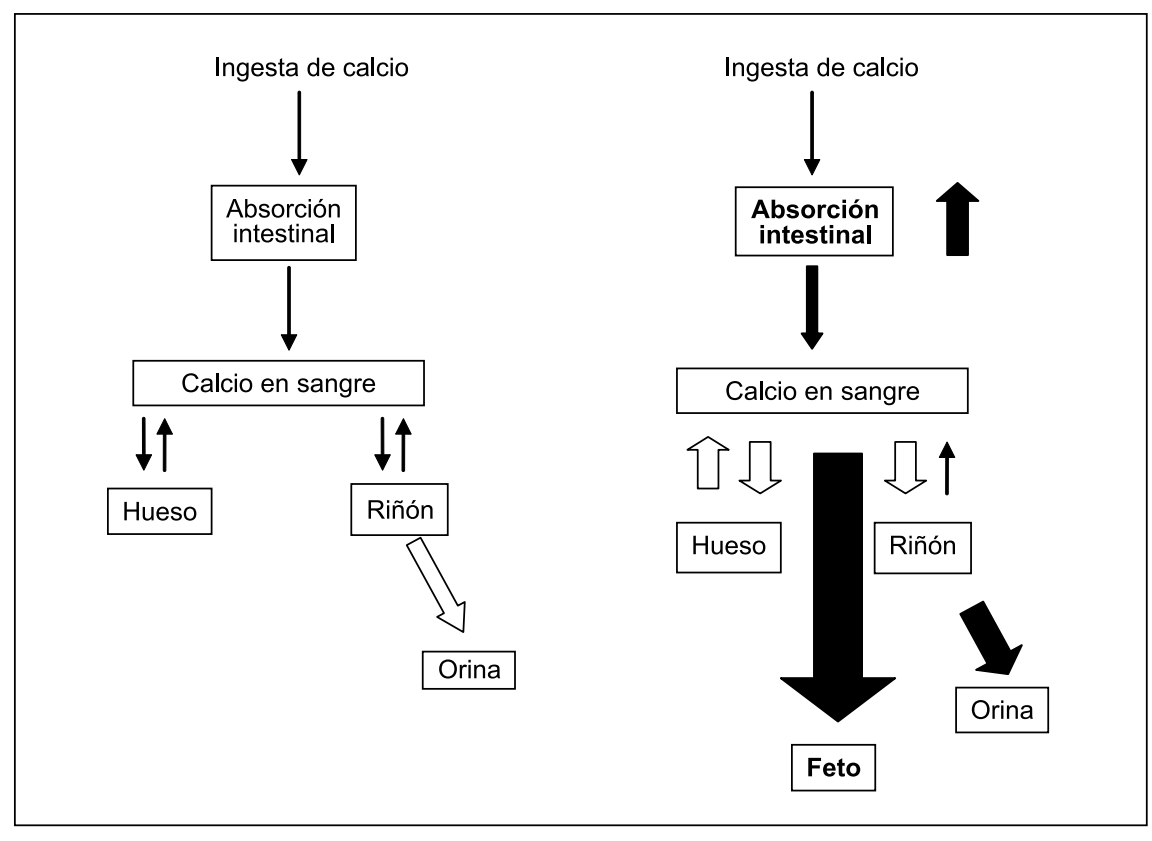

Figura 1. Metabolismo mineral óseo durante la gestación.

Durante al gestación, los mecanismos adaptativos frente a la demanda creciente de calcio, incluyen la elevación de los niveles de la $1,25(\mathrm{OH}) 2 \mathrm{D} 3$ que incrementa la absorción intestinal de calcio. A su vez, por efecto de diferentes cambios hormonales, la tasa de remodelamiento óseo se incrementa y representa otra fuente de calcio disponible para el desarrollo del esqueleto fetal.

reduce en parte el calcio ganado con el incremento en la absorción intestinal. Después del parto, la absorción de calcio y la excreción urinaria de calcio retornan a los valores previos a la gestación ${ }^{(18)}$. Figura 1.

\section{El esqueleto materno durante la gestación}

El estudio de los cambios óseos en la mujer durante la gestación ha sido motivo de continuas revisiones, a pesar de las dificultades tanto desde el punto de vista técnico como ético. Unos pocos estudios han evaluado los cambios histológicos mediante biopsia ósea en gestantes; otros han valorado los cambios en los marcadores bioquímicos del remodelamiento óseo, a pesar de que estos últimos pueden estar influenciados por diferentes factores que alterarían potencialmente los resultados durante el embarazo. El estudio de la densidad mineral ósea de la madre mediante tecnología DXA está limitado por razones éticas, básicamente asociadas con la exposición innecesaria de radiación para la madre y el feto. Sin embargo, se ha empleado otras técnicas libres de radiación, como el ultrasonido del calcáneo.
Con estudios de biopsia ósea transiliaca se ha demostrado que, durante las primeras 8 a 10 semanas de gestación, la cantidad de hueso trabecular disminuye desde un volumen relativo medio de $23 \%$ en las mujeres no gestantes hasta $16,7 \%$, primariamente debido a una disminución en el espesor trabecular y a la pérdida de la conectividad trabecular. Durante la $39^{a}$ a $40^{a}$ semana de gestación, se aprecia un incremento del número de trabéculas y una disminución de la separación trabecular, que restaura el volumen trabecular en alrededor de $24 \%{ }^{(19)}$.

Estos hallazgos sugieren que durante la gestación se produce una fluctuación en el volumen del hueso trabecular, con una pérdida temprana que se inicia después de la concepción y que posteriormente se restaura mediante la formación de nuevas trabéculas, generando un mayor número de trabéculas, aunque más delgadas. Este comportamiento bifásico, demostrado histomorfométricamente, caracterizado por un incremento inicial en la resorción ósea seguido de una formación compensatoria, se ve también reflejado por los cambios en los marcadores bioquímicos del remodelamiento óseo, que revisamos a continuación. 


\section{Marcadores bioquímicos del remode-} lamiento óseo durante la gestación

La gestación parece estar asociada a un incremento en la tasa de recambio óseo; sin embargo, es difícil predecir el efecto final de estos cambios sobre el hueso materno, solo en base a marcadores bioquímicos del remodelamiento óseo. La razón principal es que pueden estar influenciados por diferentes factores que podrían alterar los resultados, como los efectos de la hemodilución, el incremento en la tasa de filtración glomerular y la alteración en la excreción de creatinina. Asimismo, la placenta o el feto podrían estar contribuyendo en el metabolismo o degradación de algunos de estos marcadores bioquímicos ${ }^{(20)}$.

Aún con estas limitaciones, se ha informado que los marcadores de resorción ósea, en particular los productos de degradación del colágeno, como piridinolina, deoxipiridinolina y $\mathrm{N}$-telopéptido en la orina, se incrementan desde las fases tempranas hasta las etapas medias de la gestación y parecen alcanzar su pico al final del embarazo ${ }^{(21)}$.

Los marcadores de formación ósea, incluyendo la fosfatasa alcalina ósea específica o péptidos procolágenos, están disminuidos en los primeros meses de la gestación y más bien tienden a incrementarse en el tercer trimestre de la gestación. La concentración de osteocalcina se encuentra más elevada durante la gestación tardía que durante los primeros meses del embarazo, debido posiblemente a que es captada por la placenta ${ }^{(18)}$.

Estos datos parecen indicar una disociación entre la formación y la resorción ósea, con predominio de la resorción durante los dos primeros trimestres de la gestación, y una tendencia al incremento en la formación ósea hacia el final de la gestación. Sin embargo; como ya se mencionó anteriormente, el impacto sobre la densidad mineral ósea de la madre es difícil de predecir solo en base a los marcadores bioquímicos.

El incremento en la resorción ósea no se explica por los cambios en la PTH o 1,25 dihidroxivitamina $\mathrm{D}_{3}$ y más bien parecen deberse al incremento en la
PTHrp durante las etapas tempranas de la gestación, que estimula la resorción ósea en el esqueleto materno, o también a las bajas concentraciones de estrógenos en la gestación temprana ${ }^{(22)}$. El incremento en la formación ósea, descrito en etapas más avanzadas de la gestación, podría deberse a la elevación en el factor de crecimiento similar a insulina (IGF-I), que estimularía la formación ósea, o al incremento de estrógenos durante las etapas tardías del embarazo ${ }^{(23)}$.

\section{Densidad mineral ósea durante la gestación}

Existen pocos estudios clínicos que evalúen los cambios en la densidad mineral ósea de la madre durante la gestación, debido a los aspectos éticos que implican someter a radiación a una mujer gestante o al feto cuando se emplea la absorciometría dual de rayos $X$ (tecnología DXA), que es considerada al momento actual el estándar de oro para valorar la densidad mineral ósea. Por esta razón, la información disponible sobre este tópico es escasa y además sometida a otras posibles fuentes de error. Como la mayoría de estudios ha evaluado la densidad mineral ósea antes y después del parto, otra variable que potencialmente podría afectar los resultados es el tiempo transcurrido después del parto y la segunda medición de la densidad mineral ósea.

Algunos estudios de diseño caso control, empleando tecnología DXA, en los cuales se compara la densidad mineral ósea en puérperas tempranas y controles no gestantes, pareadas por edad y sexo, demuestran una disminución significativa de la densidad mineral ósea en la columna lumbar en las puérperas evaluadas entre los 3 y 5 días posteriores al parto, en elación a las no gestantes ${ }^{(24)}$.

En un estudio que valoró la densidad mineral ósea de cuerpo completo mediante tecnología DXA en 16 mujeres gestantes, se encuentra una elevación de densidad mineral ósea en sitios corticales, como antebrazos (elevación de $2,8 \%$ ) y piernas (elevación de 1,8\%), en comparación a los valores basales; mientras que los autores encuentran una reducción de la densidad mineral ósea en sitios predominantemente trabeculares, como en columna (disminución de $4,5 \%$ ) o pelvis (disminución de 3,2\%) (2). Pearson, al evaluar 60 mujeres que fueron sometidas a una densitometría ósea previa al embarazo y una segunda densitometría adquirida hasta un año después de la gestación, encuentra también una disminución de la densidad mineral ósea en un área de hueso predominantemente trabecular (columna), en porcentajes mayores de $5 \%$, y que incluso la pérdida durante la gestación alcanzó $7 \%$ en algunas pacientes. Este autor también encuentra pérdida de masa ósea en cadera total $(1,15 \%)$ y en la región trocantérica $(3,9 \%)$, pero no en cuello femoral ${ }^{(25)}$.

Black evaluó la densidad mineral ósea en 10 mujeres gestantes antes y después del parto y encontró una disminución significativa de la densidad mineral ósea en la columna (reducción de 3,5\%). En la cadera total, cuello femoral y región trocantérica, los valores de densidad mineral ósea también disminuyeron significativamente, en más de dos veces, el coeficiente de variación. En este último estudio, la reducción de la densidad mineral ósea en 3 lugares diferentes del antebrazo mostraron una reducción, pero que no llegó a ser significativa ${ }^{(21)}$.

Ritchie, en un estudio longitudinal que evaluó la densidad mineral ósea en columna lumbar, mediante tecnología DXA, no encuentra cambios en los valores de densidad mineral ósea después de la gestación, en relación a los valores previos a la gestación. Sin embargo, cabe mencionar que en este estudio se excluyó a las mujeres con un consumo pobre de calcio (menor de $800 \mathrm{mg} /$ día), y el consumo diario de calcio -excluyendo el suplemento- en las gestantes evaluadas fue elevado (en promedio $1171 \mathrm{mg} /$ día). Es importante también tomar en cuenta que en este estudio en particular, el consumo promedio de calcio se incrementó en $296 \pm 314 \mathrm{mg} /$ día durante la gestación, en comparación con la ingesta previa al embarazo, lo cual podría haber influenciado la tasa de pérdida de densidad mineral ósea de la madre. Este autor concluye que, en mujeres bien nutridas, la demanda de calcio para la mineralización del esquele- 
to fetal parece no afectar negativamente el hueso materno ${ }^{(26)}$.

Kaur reclutó a un grupo de mujeres que estaban a la espera de gestar y las sometió antes de la concepción a una densitometría ósea de columna y cadera. Durante el seguimiento, 42 mujeres resultaron embarazadas y se les repitió una segunda densitometría ósea dentro de las dos semanas posteriores al parto. Se empleó como grupo control a $32 \mathrm{mu}$ jeres que no llegaron a gestar y a las que se les repitió una segunda densitometría ósea 12 a 24 meses después ${ }^{(27)}$. En las pacientes no gestantes, Kaur no encontró diferencia significativa entre los valores obtenidos entre la primera y la segunda densitometría. Sin embargo, en el grupo de gestantes encontró una disminución de la densidad mineral ósea en la densitometría después del parto, en todos los lugares medidos, aunque esta diferencia fue significativa solo en la región intertrocantérica, una región de interés en la cadera con una elevada proporción de hueso trabecular ${ }^{(27)}$.

More evaluó a un grupo de 38 mujeres, las cuales fueron sometidas a evaluación de la densidad mineral ósea en columna lumbar, radio distal y radio ultradistal, dentro de los 3 meses previos a la gestación y seriadamente después del parto. Este autor encontró disminución significativa de la densidad mineral ósea después del parto en la columna lumbar (disminución de $2,1 \%$ ) y en menor proporción en radio distal y ultradistal $(p<0,001)$. Los autores concluyeron que el calcio requerido para el crecimiento fetal proviene del hueso trabecular y cortical de la madre, durante la gestación ${ }^{(28)}$.

Debido a las limitaciones en el empleo de la valoración de la densidad mineral ósea mediante tecnología DXA durante el embarazo, se ha empleado otras técnicas no invasivas y libres de radiaciones, como el ultrasonido cuantitativo de calcáneo. Esta técnica emplea una onda mecánica de frecuencia no audible (entre los $20 \mathrm{~Hz}$ y 100 $\mathrm{MHz}$ ), la cual es transmitida a través del hueso y, a medida que la energía de la onda de ultrasonido atraviesa el tejido óseo, el sonido interactúa con la malla trabecular y cortical del hueso, vibra en una microescala, de manera que la forma, la intensidad y la velocidad del sonido son progresivamente alteradas durante su pasaje ${ }^{(29)}$.

Mediante el ultrasonido cuantitativo de calcáneo, el tejido óseo puede ser caracterizado por dos parámetros: la velocidad de propagación de la onda de ultrasonido (SOS, expresado en $\mathrm{m} / \mathrm{s}$ ) y la atenuación de la intensidad de la onda de ultrasonido (BUA, expresada en $\mathrm{dB} / \mathrm{MHz}$ ). La evaluación de estos parámetros permite deducir las propiedades mecánicas del hueso, las cuales a su vez son importantes determinantes de la rigidez y resistencia frente a cargas y el subsiguiente riesgo de fractura ${ }^{(28)}$.

En algunos estudios longitudinales que valoran de manera seriada los parámetros de ultrasonido de calcáneo o de falanges a lo largo del embarazo, se ha demostrado una disminución tanto de la velocidad de transmisión del sonido (SOS) como la atenuación de la banda de ultrasonido (BUA) y de los parámetros compuestos, como el Índice de Rigidez (stiffness) o el Índice Cuantitativo de Ultrasonido durante la gestación ${ }^{(30,31)}$. La disminución de estos parámetros ha sido más notoria en el tercer trimestre de embarazo, justamente en el periodo de tiempo en el cual el requerimiento de calcio de parte del feto es mayor ${ }^{(32,33)}$.

Recientemente, evaluamos mediante ultrasonido de calcáneo a una población de 100 mujeres gestantes en la ciudad de Lima. Encontramos una disminución progresiva de la densidad mineral ósea durante todo el embarazo, pero más pronunciada durante el segundo trimestre de gestación. La correlación inversa entre los valores del SOS, BUA y del coeficiente stiffness en relación a la semana de gestación fue de $r=-0,264$ para el SOS, $r=-0,046$ para el BUA y $r=-0,16$ para el coeficiente de rigidez (stiffness). Entre los parámetros del ultrasonido, el SOS fue el más sensible al cambio y encontramos una disminución estadísticamente significativa entre los valores del SOS en relación a la semana de gestación (ver Figura 2) $(p=0,04)$ (34).

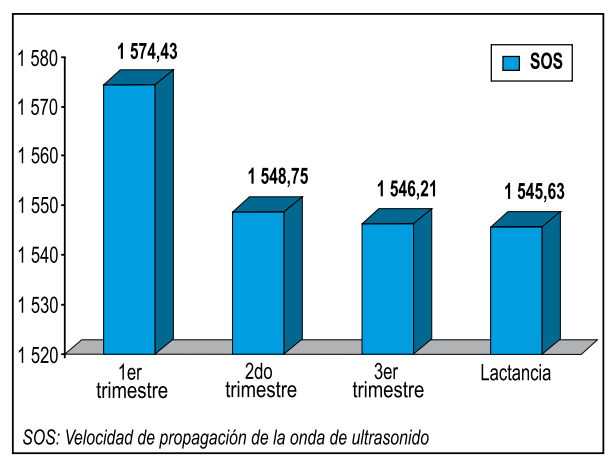

Figura 2. Cambios en la velocidad de transmisión del ultrasonido en una muestra de 100 gestantes evaluadas en el Hospital Materno-Infantil San Bartolomé de Lima.

La velocidad de transmisión del ultranido (SOS en metros/seg) disminuye en el segundo trimestre en relación al primer trimestre; y estos valores se mantienen por debajo de los valores encontrados en el primer trimestre, inclusive durante el periodo de lactancia materna.

\section{Efectos de la deficiencia de calcio sobre el hueso materno durante la gestación.}

Los efectos de la ingesta deficiente de calcio sobre el metabolismo mineral óseo y el hueso materno han sido evaluados mediante marcadores bioquímicos del remodelamiento óseo y ultrasonido de calcáneo. La mayoría de estudios parece indicar que la ingesta deficiente de calcio durante la gestación se asocia con un incremento en la tasa de remodelamiento óseo materno, lo cual tendría un efecto deletéreo sobre el esqueleto materno; y más bien, el suplemento adecuado de calcio parece relacionarse con una disminución de la tasa de remodelamiento óseo y con una menor pérdida de hueso en la madre.

Zeni, en un estudio longitudinal, diseñado para valorar el remodelamiento óseo durante el segundo y tercer trimestres del embarazo, encuentra una correlación negativa entre el incremento sérico de marcadores bioquímicos del remodelamiento óseo (NTX, CTX $\beta$ y fosfatasa alcalina ósea) y el consumo de calcio. Este autor encuentra además que la pobre ingesta de calcio es uno de los factores que parece favorecer el incremento en la tasa de remodelamiento óseo; y concluye que el suplemento adecuado de calcio durante la gestación sería beneficioso para evitar la pérdida de masa ósea a partir del esqueleto materno ${ }^{(35)}$.

Evaluando a 31 gestantes mexicanas durante el tercer trimestre del embarazo, 
Janakiraman demostró que el suplemento de $1200 \mathrm{mg}$ de calcio diarios, durante 10 días, se asociaba con una reducción significativa en los valores de NTX en orina, en cerca de $90 \%$ de las gestantes. Los autores concluyen que el suplemento de calcio podría reducir la tasa de remodelamiento óseo en el tercer trimestre de gestación, en relación a las mujeres que no reciben este suplemento ${ }^{(36)}$.

Javaid encuentra una disminución significativa de los parámetros de ultrasonido de calcáneo durante la gestación; $y$, a pesar de no encontrar una asociación global entre la ingesta de leche y los valores de ultrasonido de calcáneo, demuestra una menor disminución de la velocidad de transmisión del ultrasonido (SOS) en las mujeres con mejor ingesta de leche $(r=0,32 ; p<0,001)$. Más aún, las mujeres que recibieron suplementos nutricionales hasta el tercer trimestre de gestación presentaron mayores valores en la atenuación de la banda de ultrasonido (BUA) durante la gestación tardía $(p=$ $0,001)^{(37)}$.

Otros estudios, empleando la misma técnica de ultrasonido de calcáneo, demuestran también una mayor disminución en la velocidad de propagación del ultrasonido en mujeres con ingesta pobre de calcio, en relación a aquellas con una ingesta elevada ${ }^{(38)}$. Esta pérdida ósea valorada parece ser más pronunciada en adolescentes gestantes, en relación a gestantes adultas ${ }^{(31)}$.

Las evidencias presentadas a partir de diferentes estudios que evalúan los cambios en el metabolismo mineral óseo durante la gestación, parecen sugerir que la ingesta pobre de calcio puede afectar negativamente la masa ósea de la madre; y, contrariamente, el incremento en la ingesta de calcio reduce al menos en cierto grado esta pérdida ${ }^{(39)}$. Sin embargo, en diferentes poblaciones, el consumo de calcio promedio diario durante esta etapa de la vida de la mujer es bajo (< $500 \mathrm{mg}$ al día $)^{(12)}$, a pesar de que las recomendaciones y guías de consenso plantean que el consumo diario promedio de calcio durante la gestación y la lactancia materna debe de ser superior a los 1200 o $1500 \mathrm{mg}$ al día ${ }^{(40)}$.

\section{Efectos del suplemento de calcio en la madre sobre el esqueleto fetal}

El concepto de la modificación a nivel intrauterino del riesgo de osteoporosis en la edad adulta parece sustentarse sobre cierta base epidemiológica que relaciona el estatus nutricional, la actividad física y el tabaquismo en la madre con la masa ósea en los niños ${ }^{(41,42)}$. En modelos animales, se ha demostrado un retardo del crecimiento fetal asociado a dietas bajas en calcio y que el suplemento de calcio es crítico en mejorar el desarrollo esquelético del feto cuando la ingesta está disminuida ${ }^{(43)}$.

Koo valoró el efecto del suplemento de calcio durante la gestación sobre la mineralización del feto, en un grupo de 256 gestantes, antes de las 22 semanas de gestación, y les suplementó 2 gramos de calcio elemental o placebo hasta el parto. El autor evaluó la densidad mineral ósea en cuerpo completo y en columna lumbar en los neonatos, durante la primera semana de vida, y encontró que el contenido mineral óseo en cuerpo completo en los infantes fue significativamente mayor en los infantes nacidos de madres que recibieron suplemento de calcio en el quintil más bajo (menos de $600 \mathrm{mg}$ de calcio), en relación a las que recibieron placebo $(p<0,05)$; pero no encontraron diferencia en el contenido mineral óseo de los infantes nacidos de madres que recibían la cantidad adecuada de calcio, a despecho del suplemento de calcio ${ }^{(44)}$.

En un estudio de seguimiento prolongado, que incluyó a 797 mujeres hindúes gestantes, diseñado para evaluar el efecto del consumo de calcio durante la gestación sobre la densidad mineral ósea de los hijos, valorada al cumplir los 6 años de edad, Ganpule encontró que los niños nacidos de madres que durante la gestación tuvieron una mayor frecuencia de consumo de alimentos ricos en calcio tenían una mejor densidad mineral ósea en columna y cuerpo completo a los 6 años de edad, en relación a los niños cuyas madres tuvieron un consumo pobre de calcio durante la gestación. Este autor concluye que el consumo de calcio es uno de los factores modificables de la dieta, que parece favorecer la salud ósea en el niño ${ }^{(45)}$.

\section{Comentarios finales}

El metabolismo óseo durante la gestación ha sido motivo de diferentes revisiones, focalizadas básicamente en su probable impacto sobre el esqueleto materno y fetal. El incremento de la demanda del calcio, debido al crecimiento del esqueleto fetal durante la gestación, requiere de un aporte adicional de este mineral, el cual en condiciones de deprivación o de pobre ingesta sería removido del esqueleto materno, llevando a una pérdida de masa ósea en la madre. Después de la gestación, la lactancia materna continuaría demandando mayor aporte de calcio para concentrarlo en la leche materna; y se calcula que en esta etapa de la vida la madre pierde adicionalmente entre 2\% y $7 \%$ de masa ósea en columna lumbar y cuello femoral ${ }^{(1)}$.

Sin embargo, la masa ósea perdida durante la gestación y la lactancia materna se tiende a recuperar después del destete; y la velocidad de recuperación de la masa ósea materna se relaciona con la duración de la lactancia materna y la duración del periodo de amenorrea posparto ${ }^{(46)}$. A pesar de que la tasa de recuperación de la masa ósea está influenciada por diferentes factores, requiere de un periodo de tiempo considerable para que retorne a los valores previos a la gestación. Al igual que durante la gestación y la lactancia materna, la recuperación de la masa ósea después del destete es más rápida en las mujeres que reciben aporte adecuado de calcio en relación a aquellas que no lo reciben ${ }^{(47)}$.

Las consecuencias de la gestación y la lactancia sobre el esqueleto materno nos llevan finalmente a tomar en cuenta dos aspectos importantes. El primero es el impacto sobre el pico de masa ósea en la madre. Algunas evidencias antropológicas parecen sugerir que el efecto de las gestaciones múltiples y periodos de lactancia prolongada podrían tener un efecto deletéreo sobre el pico de masa ósea de la madre y un mayor riesgo de osteoporosis y fracturas en etapas posteriores de la vida ${ }^{(48)}$. La gestación es parte 
de la vida reproductiva de toda mujer y la lactancia materna es claramente benéfica para el niño, pero ambas pueden ser deletéreas para el esqueleto materno. De manera que, el segundo aspecto que quisiéramos remarcar es que durante estas dos etapas el suplemento adecuado de calcio a la madre gestante debe de formar parte de la rutina de los cuidados prenatales y posnatales.

Las evidencias revisadas en estudios clínicos sobre el papel del aporte adecuado de calcio durante la gestación y la lactancia materna, en reducir la tasa de remodelamiento óseo y evitar la pérdida de masa ósea, hacen necesario que se considere como una prioridad el aporte adecuado de calcio, conjuntamente con otros nutrientes, durante estas etapas de la vida.

\section{REFERENCIAS BIBLIOGRÁFICAS}

1. Kalkwarf HJ. Calcium in pregnancy and lactation. En: Weaver CM, Heaney RP, Raisz LG. Calcium in Human Health. Totowa, NJ; Humana Press Inc. 2006:297-309.

2. Naylor KE, lqbal P, Fledelius C, Fraser RB, Eastell R. The effect of pregnancy on bone density and bone turnover. J Bone Min Res. 2000;15:129-37.

3. Namgung R, Tsang RC. Bone in the pregnant mother and newborn at birth. Clin Chimica Acta. 2003;333:1-11.

4. Prentice A. Calcium in pregnancy and lactation. Annu Rev Nutr. 2000;20:249-72.

5. Hamaoui E, Hamaoui M. Nutritional assessment and support during pregnancy. Gastroenterol Clin N Am. 2003;32:59-121.

6. Kerr C, Loken HF, Glendening MB, Gordon CS, Page EW. Calcium and phosphorus dynamics in pregnancy. Am J Obstet Gynecol. 1962;83:2-8.

7. Kovacs ChS, Kronenberg HM. Maternal-fetal calcium and bone metabolism during pregnancy, puerperium, and lactation. Endocr Rev. 1997;18:832-72.

8. Reynolds WA, Williams GA, PitkinRM. Calcitropic hormone responsiveness during pregnancy. Am J Obstet Gynecol. 1981;139:855-62.

9. Verhaeghe J, Bouillon R. Calciotropic hormones during reproduction. J Steroid Biochem Mol Biol. 1992;41:469-77.

10. Gao P, Scheibel S, D'Amour P, John MR, Rao $\mathrm{SD}$, Schmidt-Gayk $\mathrm{H}$, et al. Development of a novel immunoradiometric assay exclusively for biologically active whole parathyroid hormone 1-84: implications for improvement of accurate assessment of parathyroid function. J Bone Miner Res. 2001;16:605-14.

11. Singh HJ, Mohammed NH, Nila A. Serum calcium and parathormone during normal pregnancy in
Malay women. J Matern Fetal Med. 1999;8:95100.

12. Vargas Zapata CL, Donangelo CM, Woodhouse LR, Abrams SA, Spencer EM, King JC. Calcium homeostasis during pregnancy and lactation in Brazilian women with low calcium intakes: a longitudinal study. Am J Clin Nutr. 2004;80:41722.

13. Cross NA, Hillman LS, Allen SH, Krause GF, Vieira NE. Calcium homeostasis and bone metabolism during pregnancy, lactation, and postweaning: a longitudinal study. Am J Clin Nutr. 1995;61:51423.

14. Ogueh 0, Khastgir G, Abbas A, Jones J, Nicolaides KH, Studd JW, et al. The feto-placental unit stimulates the pregnancy-associated increase in maternal bone metabolism. Hum Reprod. 2000;15:183437.

15. Cornish J, Callon KE, Nicholson GC, Reid IR. Parathyroid hormone-related protein-(107-139) inhibits bone resorption in vivo. Endocrinology. 1997;138:1299-304.

16. Kronenberg HM. PTHrP and skeletal development. Ann N Y Acad Sci. 2006;1068:1-13.

17.0'Brien K0, Nathanson MS, Manzini J, Witter FR. Calcium absorption is significantly higher in adolescents during pregnancy than in the early postpartum period. Am J Clin Nutr. 1998;67:693701.

18. Salle BL, Delvin EE, Lapillonne A, Bishop NJ, Glorieux FH. Perinatal metabolism of vitamin D. Am J Clin Nutr. 2000;71 (suppl 1):1317S-24S.

19. Shahtaheri SM, Aaron JE, Johnson DR, Purdie DW. Changes in trabecular bone architecture in women during pregnancy. Br J Obstet Gynaecol. 1999;106:432-8.

20. Kovacs ChS, Fuleihan GE. Calcium and bone disorders during pregnancy and lactation. Endocrinol Metab Clin N Am. 2006;35:21-51.

21. Black A, Topping J, Dirham B, Farquharson R, Fraser A. A detailed assessment of alterations in bone turnover, calcium hemostasis, and bone density in normal pregnancy. J Bone Miner Res. 2000;15:557-64.

22. Martin TJ, Moseley JM. Parathyroid hormone-related protein. En: DeGroot LJ, editor. Endocrinology. Philadelphia: Saunders; 1995. p. 967-77.

23. Nilsson BE. Parity and osteoporosis. Surg Gynecol Obstet. 1969;129:27-8.

24. Honda A, Kurabayashi T, Yahata T, Tomita M, Takakuwa K, Tanaka K. Lumbar bone mineral density changes during pregnancy and lactation. Int J Gynecol Obstet. 1998;63:253-8.

25. Pearson D, Kaur M, San P, Lawson N, Baker P, Hosking. Recovery of pregnancy mediated bone loss during lactation. Bone. 2004;34:570-8.

26. Ritchie LD, Fung EB, Halloran BP, Turnlund JR, Van Loan MD, Cann CE, et al. A longitudinal study of calcium homeostasis during human pregnancy and lactation and after resumption of menses. Am J Clin Nutr. 1998;67:693-701.

27. Kaur M, Pearson D, Godber I, Lawson N, Baker $\mathrm{P}$, Hoskinga D. Longitudinal changes in bone mineral density during normal pregnancy. Bone. 2003;32:449-54.

28. More C, Bettembuk P, Bhattoa HP, Balogh A. The effects of pregnancy and lactation on bone mineral density. Osteoporos Int. 2001;12:732-7.

29. Vidal L. Ultrasonido cuantitativo. Bases y Principios de la Densitometría Ósea. Lima, CEDOR/EDIYUSA; 2005. p. 104-13.

30. Paparella P, Giordino R, Maglione A. Maternal ultrasound bone density in normal pregnancy. Clin Exp Obstet Gynecol. 1995;22:268-78.

31. Sowers MF, Scholl PT, Harris I, Jannausch M. Bone loss in adolescent and adult pregnant women. Obstet Gynecol. 2000;96:189-93.

32. Gambacciani M, Spinetti A, Gallo R, Cappagli $B$, Teti GC, Facchini V. Ultrasonographic bone characteristics during normal pregnancy: Longitudinal and cross-sectional evaluation. Am J Obstet Gynecol. 1995;173:890-3.

33. Yamaga A, Taga M, Minaguchi H. Changes in bone mass as determined by ultrasound and biochemical markers of bone turnover during pregnancy and puerperium: A longitudinal study. J Clin Endocrinol Metab. 1996;81:752-6.

34. Vidal L, Santivañez V. Calcio durante la gestación y lactancia materna (conferencia). III Congreso Bolivariano del Climaterio y Menopausia. VII Congreso Peruano de Climaterio. Lima, Perú. 6 al 8 de Julio de 2008.

35.Zeni SN, Ortela SL, Lazzari A, López L, Suarez M, Di Gregório S, et al. Interrelationship between bone turnover markers and dietary calcium intake in pregnant women: a longitudinal study. Bone. 2003;33:606-13.

36. Janakiraman V, Ettinger A, Mercado-Garcia A, Hu $H$, Hernandez-Avila M. Calcium supplements and bone resorption in pregnancy. A randomized crossover trial. Am J Prev Med. 2003;24:260-4.

37. Javaid MK, Crozier SR, Harvey NC, Taylor P, Inskip HM, Godfrey KM, et al. Maternal and seasonal predictors of change in calcaneal quantitative ultrasound during pregnancy. I Clin Endocr Metab. 2005;90:5182-7.

38. Aguado F, Revilla M, Hernandez ER, Menendez M, Cortez-Prieto J, Villa LF, et al. Ultrasonographic bone velocity in pregnancy: a longitudinal study. Am J Obstet. Gynecol. 1998;178:1016-21.

39. Thomas M, Weisman SM. Calcium supplementation during pregnancy and lactation: Effects on the mother and the fetus. Am J Obstet Gynecol. 2006:194:937-45.

40.Standing Committee on the Scientific Evaluation of Dietary Reference Intakes. Washington, D.C.: National Academy Press ;1997.

41. Jones G, Riley M, Dwyer T. Maternal smoking during pregnancy, growth and bone mass in prepubertal children. J Bone Miner Res. 1999;14:146-51.

42. Tobias JH, Steer CD, Emmett PM, Tonkin RJ, Cooper C, Ness AR. Bone mass in childhood is related to maternal diet in pregnancy. Osteoporos Int. 2005;16:1731-41.

43. Lima MS, Kallfelz F, Krook L, Nathanielsz PW. Humeral skeletal development and plasma 
constituent changes in fetuses of ewes maintained on a low calcium diet from 60 days of gestation. Calcif Tissue Int. 1993;52:283-90.

44. Koo WW, Fraccp MBBS, Walters J, Esterlitz J, Levine RJ, Bush AJ, et al. Maternal calcium supplementation and fetal bone mineralization. Obstet Gynecol. 1999;94:577-82.

45. Ganpule A, Yajnik CS, Fall CHD, Rao S, Fisher DJ, Kanade $A$, et al. Bone mass in Indian childrenrelationships to maternal nutritional status and diet during pregnancy: the Pune Maternal Nutrition Study. J Clin Endocrinol Metab. 2006;91:29943001.

46. Polatti F, Capuzzo E, Viazzo F, Colleoni R, Klersy C. Bone mineral changes during an after lactation. Obstet Gynecol. 1999;94:52-6.

47. Kalkwarf HJ, Specker BL, Bianchi DC, Ranz J, Ho $M$. The effect of calcium supplementation on bone density during lactation and after weaning. N Engl J Med. 1997;337:523-8.

48. Agarwal SC, Dumitriu M, Tomlinson GA, Grynpas MD. Medieval trabecular bone architecture: The Influence of age, sex, and lifestyle. Am J Phys Anthropology. 2004;124:33-44.

Manuscrito recibido el 28 de setiembre de 2008 y aceptado para publicación el 25 de setiembre de 2008.

Correspondencia:

Dr. Luis Vidal Neira

Av. Paseo de la República 3691. Dpto. 201.

Lima 27, Perú.

Correo-e: Ifvidaln@hotmail.com 